\title{
XXXIV.
}

Aus der Königl. chirurgischen Universitätsklinik zu Kiel. (Prof. Helferich.)

\section{Zum Mechanismus der Luxation im unteren Radioulnargelenk.}

Von

Dr. F. Wilh. Baum, Assistenzarzt der Klinik.

(Mit 2 Abbildungen.)

Am 30. April d. J. suchte der 44jährige Arbeiter Friedrich H. aus Bordesholm die Hülfe der Kgl. Chirurg. Klinik zu Kiel auf. Er gab an, er sei am Morgen dieses Tages beim Steinaufladen verletzt worden. Gerade, als er in gebückter Stellung mit beiden Händen einen Stein umfasste, um ihn zu heben, fiel ein anderer ca. 1 Centner schwerer Stein aus beträchtlicher Höhe herab und traf $\mathrm{H}$. gegen den rechten Unterarm. Die anfänglichen Schmerzen waren nur gering, jedoch war H. ausser Stande, die rechte Hand zu bewegen. Eine kleine Wunde am Handrücken blutete stark. Der hinzugezogene Arzt legte einen Nothverband an und übersandte den Patienten der Klinik.

Bei dem kräftig gebauten und im Uebrigen gesunden Manne ist der rechte Unterarm in seinem unteren Drittel merkwürdig deformirt; er ist leicht gesehwollen, in der Fläche auffallend schmal, während der Tiefendurchmesser deutlich verlängert ist. Auf dem Dorsum besteht zwischen Radiusepiphyse und Ulna eine beträchtliche Niveaudifferenz zu Gunsten der ersteren; umgekehrt findet sich volarwärts auf der ulnaren Seite eine starke Prominenz; ein fester Zusammen hang zwischen dieser und dem Ulnaschaft ist unschwer nachzuweisen. Die Vorwölbung lässt die Form des Ulnaköpfehens erkennen, obwohl der so charakteristische Styloidfortsatz nicht abgetastet werden kann; auch an seinem normalen Platze ist er nicht fühlbar. Statt dessen findet sich an dieser Stelle ein wie mit dem Locheisen geschlagener Defect, der jedoch nur die obersten Gewebsschichten betrifft; die ein- 
geführte Sonde dringt nicht tief vor und stösst nirgends auf Knochen. Die ulnare Seite des Vorderarmes ist sehr schmerzhaft. Crepitation ist hier nicht nachweisbar. Die normalen Contouren des Radius sind überall gut abtastbar, auch sonst fehlt am Radius jedes Zeichen einer Fractur. Die Hand steht in leichter Supination und Abduction; die Bewegungen im Handgelenk sind sehr behindert, Pro- und Supination

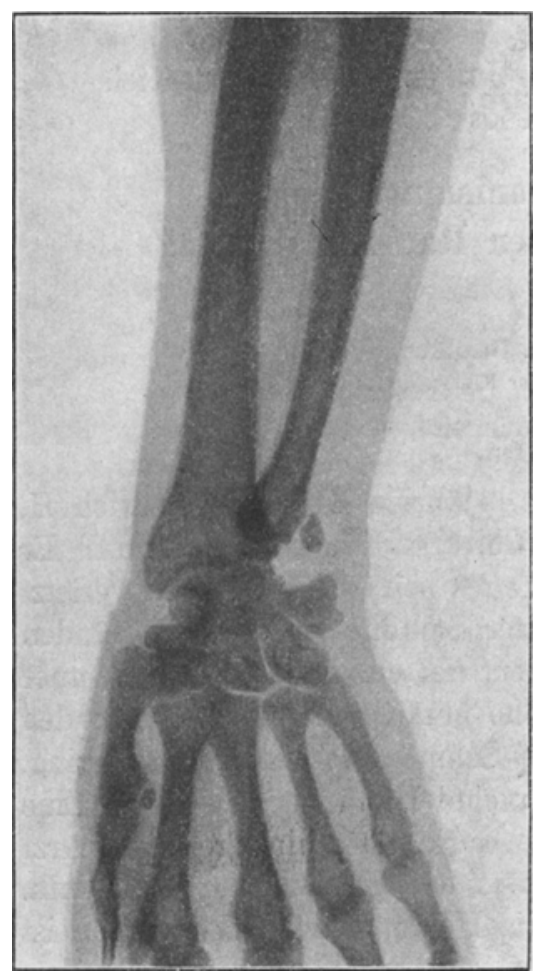

I’ig. 1.

Aufnahme von vorne.

1. Radiale Verschiebung der Ulna.

2. Absprengung des Proc. styloideus ulnae.

3. Radius intact.

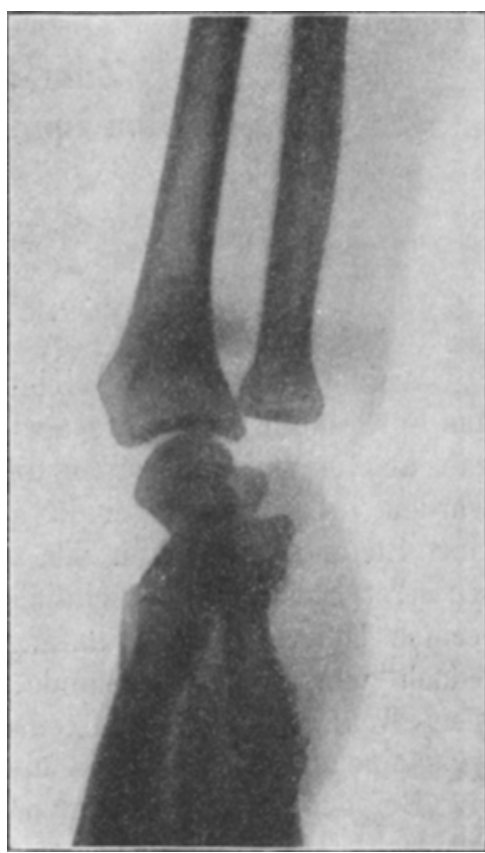

Fig. 2 .

Seitliche Aufnahme.

Es tritt hier deutlich die volare Dislocation der Ulna hervor. Der Radius ist intact.

sind aufgehoben, Volar- und Dorsalflexion in sehr beschränktem Maasse ausführbar. Actives Beugen der Finger ist nur wenig möglich, passives äusserst schmerzhaft.

Das Röntgenbild zeigt von der Fläche aufgenommen eine radiale Verschiebung der Ulna, sie kreuzt sich mit dem völlig intacten Radius; von der Seite aufgenommen, sieht man das volare Vorspringen der 
Ulna; auf Bild I tritt eine Absprengung des Processus styloideus klar hervor.

Die Reposition gelang in Narkose sofort ohne jede Schwierigkeit Bei Extension der Hand mit Gegenzug am Ellenbogen, dann Radialflexion und schliesslich starker Beugung sprang das Ulnaköpfchen unter hörbarem Knacken in seinen alten Platz ein. Die Fixirung geschah mit Schede'scher Radiusschiene für die linke Hand, $d . h$. also in Radialflexion und Beugung. Nach achttägigem Verbande steht der Arm gut, er ist schmerzlos. Die Ulna zeigt keinerlei Neigung, in die luxirte Stellung zurückzuweichen. Unter täglich mehrstiindigen warmen Bädern wurde nach weiteren acht Tagen die normale Beweglichkeit der Hand wieder erreicht. Die Stellung der Ulna ist jetzt insofern etwas übercorrigirt, als sie ein wenig mehr dorsalwärts als normal steht; sie lässt sich leicht federnd zurückdrücken. Die Röntgenaufnahme bestätigt diesen palpatorischen Befund.

Die in unserem Falle sehr charakteristischen Symptome, Verkleinerung des Quer-, Verbreiterung des Tiefendurchmessers am untersten Abschnitt des Vorderarms, sowie die Verlagerung der normalen hinteren Ulnaprominenz an die Vorderseite des Handgelenks deuteten ohne Weiteres auf eine Luxation im unteren Radioulnargelenk und zwar, da die Vorwölbung sich vorne befand, auf eine volare Dislocation. Als Complication tritt ein Bruch des Griffelfortsatzes der Ulna hinzu.

Aetiologisch ist directe Gewalt, bedingt durch einen fallenden Stein, der den in Flexion stehenden Vorderarm trifft, heranzuziehen. Die Reposition bestand in Extension, Radialflexion und Beugung. Heilung in guter Stellung und mit normaler Function trat nach 14 Tagen ein. Es hat sogar eine Uebercorrection der Luxationsstellung stattgefunden in Gestalt einer ganz geringen dorsalen Verschiebung des Ulnaköpfchens; dies ist durch die Absprengung des Proc. styloideus und die hieraus resultirende Lockerung der Bandverbindung (Lig. subcruentum) von Ulna und $O$ s triquetrum zu erklären.

Da selbst namhafte Chirurgen sich nicht erinnern, eine derartige Verletzung, d. h. die nicht mit Radiusfractur combinirte Luxation je gesehen zu haben, und auch die Casuistik nur relativ wenige Fälle aufweist, veranlasste mich Herr Geh. Rath Helferich, dem ich für die Anregung zu dieser Arbeit meinen aufrichtigen Dank sage, unsere Beobachtung mitzutheilen. In der mir zugänglichen Literatur nach der Entdeckung der Röntgenstrahlen fand ich keinen ähnlichen Fall beschrieben.

Ich möchte im Anschluss an die Krankengeschichte kurz auf die 
Entstehungsweise dieser Luxation, wie sie sich klinisch und experimentell ergiebt, eingehen. Die erste grundlegende Arbeit über die Verrenkung im unteren Radioulnargelenk stammt von Malgaigne; er hat die in der Literatur zerstreuten casuistischen Beiträge kritisch verwerthet und aus diesen und eigenen Beobachtungen uns ein scharf abgegrenztes Krankheitsbild gegeben. Auf Grund seiner klinischen Untersuchungen kommt er bezüglich der Aetiologie dieser Verletzung zu dem Schluss, dass die volare Luxation durch extreme Supination, die dorsale durch Pronation der Hand zu Stande komme.

Nach ihm hat dann Tillmanns sich mit dem gleichen Gegenstand befasst und unter Heranziehung der von Malgaigne angeführten Fälle über 16 volare und 19 dorsale Luxatjonen im unteren Radioulnargelenk berichtet. Auch er bezeichnet als Ursache für die vordere Luxation Supination, für die hintere maximale Pronation. Liese Angaben sind in sämmtliche Lehrbücher übernommen worden.

Als ich gelegentlich meiner Beobachtung den Mechanismus dieser Verletzung studirte, schien es mir, als liesse sich die heutige Lehre mit den anatomischen Verhältnissen nicht recht in Einklang bringen. Ich prüfte daher nochmals die Frage der Entstebung nach und lasse hier kurz die Ergebnisse meiner Uutersuchung folgen.

Die Rotationsbewegung der Hand geschieht nach Henke durch Drehung des Radius um eine Axe, welche dureb sein oberes Ende und das untere der Ulna geht; dabei dreht sich der Radius im Ellenbogen um sich selbst, an der Hand um die Ulna; hier im Gegensatz zur Ulna in fester Verbindung mit der Hand. Da, wo beide Vorderarmknochen sich kreuzen, liegt der Stützpunkt, über den Radius wie Ulna nach der einen oder der andern Richtung herausgehebelt werden können; die Ulna aus der unteren, der Radius aus seiner oberen Gelenkverbindung, je nachdem das eine oder das andere Gelenk widerstandsfähiger ist. Begünstigt wird dabei die Luxation des unteren Ulnarandes durch den grossen Spielraum, der sich dem Capitulum ulnae in dem schlaffen Kapselsacke der Membrana saceiformis bietet, während es andererseits durch keines der zahlreichen Bänder der Hand fixirt wird.

Als ätiologisches Moment kommt hierbei in erster Linie, wie die bisherigen Beobachtungen zeigen, die directe Gewalt in Betracht. Die Gewalt trifft entweder direct die Ulna oder aber den Radius; da letzterer fest mit dem Carpus verwachsen, wird nicht er vorgestossen, sondern vielmehr die leicht bewegliche Ulna in der entgegengesetzten Richtung heraus gedrängt. Am häufigsten ist dies wohl der Fall, wenn der Vorderarm mit der Ulna aufliegt und der Radius von vorne oder 
hinten getroffen wird. Um die indirecte Entstehungsweise experimentell zu priffen, habe ich die Luxation durch forcirte Supination und Pronation an Leichen zu erzengen versucht. Ich habe meine Versuche an der intacten Extremität, am Arm mit präparirten Gelenken und am Scelett angestellt. Ich will vorausschicken, dass es mir nicht gelungen, eine ausgesprochene Luxation der Ulna hervorzurufen. Ich finde mich da in Uebereinstimmung mit $\mathrm{H} \ddot{o n i g s s h m i d t ~ u n d ~ B o n n e t . ~}$ die zu einander gleichen Resultaten bei ihren Experimenten gelangt sind. Hönigschmidt sah in 20 Versuchen über gewaltsame Pronation der Hand gar keine Störungen im unteren Radioulnargelenk auftreten. Erst an einem präparirten Arm gelang es ihm, die Bewegung soweit zu forciren, dass das vordere Ende der Incisura semilunaris radii über die zum Proc. styloideus führende Kante der Ulna etwas vorbeiglitt, so dass der Radius in extremer Pronationsstellung fixirt blieb; durch eine leichte Supinationsbewegung kehrte derselbe aber sofort in seine frühere Lage zurück. Bei übermässiger Supination sah er häufig das Capitulum ulnae volarwärts hervorragen, die vordere Wand der Membrana sacriformis über demselben stark gespannt oder vertical durchtrennt. Ferner waren zuweilen der Proc. styloideus gebrochen, zuweilen die Cartilago triangularis vom Radius abgerissen. Hönigschmidt wie auch Bonnet heben jedoch ausdrücklich hervor, dass diese Dislocationen des unteren Ulnaendes niemals vollständige und dauernde waren; sobald die rotirende Gewalt zu wirken aufhörte, kehrten die Gelenktheile in ihre früheren räumlichen Beziehungen zurïck. Es fehlte also der für die Luxation charakteristische Factor, die „dauernde Verschiebung"; mit Recht bezeichnen daher beide Autoren die genannten Veränderungen nur als Distorsion im unteren Radioulnargelenk.

Bei meinen nur in geringem Umfange vorgenommenen Versuchen kam ich zu völlig übereinstimmenden Resultaten. Bei extremer Supination brach entweder der Processus styloideus ulnae $\mathrm{ab}$, während das Köpfchen an normaler Stelle verblieb, oder es riss die Membrana sacriformis an der Vorderseite vertical ein; das Köpfchen trat dabei durch den Schlitz ein wenig volarwärts beraus, ohne jedoch in dieser Stellung fixirt zu bleiben; es handelte sich auch hier nur um eine Distorsion des Radioulnargelenkes. Bei übermässiger Pronation fracturirte der Radius; es gelang mir durch diese Drehung der Hand nicht, eine Dislocation der Ulna hervorzurufen.

Kann nun überhaupt durch Pronation eine dorsale, durch Supination eine volare vollkommene Luxation zu Stande kommen?

Lässt man am scelettirten Arm die Hand eine pronirende Be- 
wegung machen und zwar so weit, dass der Radius sich dabei um $180^{\circ}$ gedreht hat, so stösst die Ulna gegen den Radius in der Mitte des Unterarmes von unten an, und damit ist, vorausgesetzt, dass der Radius intact bleibt, einer dorsalwärts gerichteten Bewegung der Ulna ein mechanisches Hinderniss gesetzt. Geht vielmehr die Hand mit dem Radius, welche wir wegen der sehr straffen Radiocarpalverbindung als ein zusammenhängendes Ganzes auffassen können, in der pronirenden Bewegung weiter, so gleitet der Carpus am Processus styloideus vorbei, und die Ulna wird nach vorne berausgehebelt. Entsprechend verhält es sich bei extremer Supination. Wird die Hand über die parallele Stellung beider Vorderarmknochen hinaus forcirt supinirt, so wird das Ulnaköpfchen durch den hinteren Rand des Sinus semilunaris radii nach hinten herausgehebelt; bei fortwirkender supinirender Gewalt muss doch jedenfalls der Carpus an die Vorderseite der Ulna treten und nicht umgekehrt diese an die Volarseite der Hand.

Es ist daher meines Erachtens schon mechanisch ganz unmöglich, dass extreme Supination zur volaren extreme Pronation zur dorsalen vollständigen Luxation führt. Wenn überhaupt auf diese indirecte Weise jene Verletzung entstehen kann, so wird das nur umgekehrt, als bisher angenommen, zu Stande kommen können. Wird nämlich die pronirende und supinirende Drehung nicht von der Hand bei fixirtem Unterarm ausgeführt, sondern werden vielmehr bei festgestellter Hand am Vorderarm Bewegungen im Sinne der Supinaton beziehungsweise Pronation gemacht, so ist es mechanisch möglich, dass durch hastige Innendrehung des Armes die Ulna dorsal, durch Aussendrehung volar hervortritt; an der Leiche ist es mir nicht gelungen, auf diesem Wege eine Dislokation zu erzielen. Klinisch beobachtet man einen derartigen Mechanismus bei der habituellen Dorsalluxation, wie sie durch die dauernd pronirende Bewegung des Wäscheausringens hervorgerufen wird; auch hier wird die Pronation nicht von der Hand ausgeführt, vielmehr ist diese der feste Punkt, um den sich der Vorderarm nach innen dreht.

Sehen wir einmal kurz die wenigen seit der Tillmanns'schen Arbeit (1874) mitgetheilten Beobachtungen von Luxation im unteren Radioulnargelenk auf die Art ihrer Entstehung hin an. In der mir zugänglichen Literatur habe ich im ganzen 12 Fälle nicht complicirter Verenkung beschrieben befunden, von denen 9 englischen und amerikanischen Zeitschriften entnommen sind, während drei der deutschen Literatur angehören. Zehnmal wurde die volare, nur zweimal die dorsale Dislocation beobachtet. Leider ist in vieleu Mittheilungen die Aetiologie nur ungenau behandelt, sodass sie nach dieser Richtung: 
nur sehwer zu verwerthen sind. Bei einzelnen gewinnt man den Eindruck, als habe die Kenntniss der Malgaigne'schen Lehre den Verfasser beim Erheben der Anamnese beeinflusst.

Ich theile hier kurz die Fälle mit:

1. Purdom berichtet Folgendes: Ein Mann war mit seiner Hand zwischen die Rollen einer Hobelmaschine gerathen, wobei auch der Vorderarm in schiefer Richtung hereingezogen wurde. Die Rollen, die eine über der anderen lagen, gaben im Augenblick etwas nach, so dass der Arm noch gerettet werden konnte. Die Untersuchung: ergab eine Höhlung an der ulnaren Seite des Handgelenkrückens, während sich vorne eine Prominenz von der Form des Processus styloideus ulnae abtasten liess.

2. Ohlemann fand bei einem Arbeiter, dem von einem Pferde der linke Vorderarm gegen die Krippe gedrängt war, die Ulna volar und radialwärts dislocirt.

3. Horrocks beschreibt die dorsale Verrenkung bei einem alten Manne, der mit seinem linken pronirten Vorderarm zwischen zwei schwere Kisten gerathen war. Bei der Untersuchung zeigte sich der Vorderarm stark geschwollen, die Hand stand in extremer Pronation; auf der Hinterseite des Radius fand sich ein Vorsprung, der mit der Ulna eng zusammenhing.

4. Kirkby-Thomas berichtet über einen jungen Mann, der, beim Löschen eines Dampfers beschäftigt, von einem herabfallenden schweren Eisenstiick getroffen wurde, das gegen die Hinterseite seines Vorderarmes aufschlug; die Ulna prominirte volar und war vor dem Radius zu fühlen.

5. Briggs sah ebenfalls auf directe Weise die Verrenkung zu Stande kommen. Eine 62jährige Frau, die vom Kopfende ihres Bettes zu dessen Fussende gehen wollte, wurde plötzlich schwindelig und fiel aufs Gesicht. Beim Versuch, sich zu halten, ergriff sie die Bettkante mit ihrer linken Hand, die so zu liegen kam, dass sie von einer darüberhängenden Rolle bedeckt wurde. Als nun der Arm mit dem Körper die Bewegung nach vorwärts mitmachte, drückte die Spitze der Rolle fest auf die Hinterseite der Ulna, diese fixirend. Dadurch wurde das Capitulum ulnae nach vorne gedrängt.

6. Weir theilt die Verletzung einer 84 jährigen Frau mit, die bei fixirtem Vorderarme an der stark supinirten Hand heftig gezogen wurde. Das Ulnaköpfchen ragte volar nahe der Mittellinie hervor.

7. Neve beobachtete die Luxation nach dem Handrücken bei einem jungen Mädchen, das eilig um eine Strassenecke lief und dabei seitwärts hinstïrzte. Sie streckte die Hand nicht abwehrend aus, son- 
dern fiel auf die Hinterfläche der gebeugten Hand. Bei der sofortigen Untersuchung fand sich die Hand flectirt und pronirt; auf dem Dorsum sah man eine beträchtliche Vorwölbung, welche die Hand nach vorne verschoben erscheinen liess. Die Ulna war dorsal luxirt und stand auf der Hinterseite des Radius.

8. Stierlin constatirte Luxatio volaris bei einem 66 jährigen Arbeiter, der einen Riemen über die Rolle einer Maschine legen wollte; dabei fasste ihn der Riemen bei der Hand und drehte seinen Arm gewaltsam nach aussen. Das Capitulum ulnae war als abnorme Prominenz volar dicht über der Handgelenkspalte sichtbar.

9. Nach Weiz verunglückte eine Frau in derselben Weise dadurch, dass ihr ein schnell herabfallendes Speisebrett, während der Vorderarm auf einem Sims ruhte, auf die Hand schlug und diese gewaltsam supinirte, was eine Luxation des Ulnaköpfehens nach vorne zur Folge hatte.

10. Makeig J ones giebt folgende Entstehungsursache an. Ein 55 jähriger Bergwerksarbeiter war an einer Bohrmaschine beschäftigt, als der Bolzen ausglitt, ihn gegen die rechte Hand traf und diese sehr stark pronirte, während der Vorderarm gleichzeitig mehr oder weniger fixirt blieb. Nach der Verletzung war die Hand gebeugt, der dem Ulnaköpfchen entsprechende Vorsprung auf der Rückseite fehlte; an seiner Stelle fand sich eine eigenthümliche Grube. Etwas distal davon fühlte man eine Vorwölbung, die an der Unterseite deutlich die Conturen der Ulna zeigte. An der Stelle, wo der Bolzen getroffen, waren die Weichtheile oberflächlich verletzt. Die Diagnose wurde auf volare Luxation im unteren Radioulnargelenk gestellt.

11. White beobachtete die gleiche Verletzung bei einem Manne, der ein Seil ausbessern wollte, das um eine Winde gelegt war; er gerieth mit seiner Hand zwischen Winde und Seil. Das Capitulum ulnae lag auf der Vorderfläche des Radius.

12. Leistend orfer lässt die von ihm beschriebene Verletzung. folgendermaassen entstehen. Ein Soldat glitt beim Voltigiren mit der rechten Hand ab, quetschte sich beim Fallen das rechte Handgelenk zwischen Voltigirbock und Brustkorb, wobei das Ulnaköpfchen dem abrollenden Körper als Hypomochlion diente. Das Capitulum ulnae stand volar vor dem Radius.

Von den 12 ausgeführten Luxationen sind nach Angabe der Beobachter fünf, darunter vier volare, eine dorsale, direct entstanden, die übrigen sieben, davon 6 volare und ebenfalls nur eine dorsale auf indirectem Wege. $\mathrm{Zu}$ den erstgerfannten gehören die Fälle von Purdom, Ohlemann, Horrocks, Kirkby-Thomas und Briggs; 
ihnen reiht sich als sechster der meinige an. In Mittheilung 1 und 2 wurde durch local wirkenden Druck die Ulna nach vorne, in Mittheilung 3 nach hinten herausgedrängt. Bei Thomas' und meiner Beobachtung war es ein schweres Gewicht (Eisenstïck, Stein), das aus beträchtlicher Höhe den Vorderarm traf und das Ulnaköpfehen volarwärts dislocirte. In Fall 5 musste die fixirte Ulna beim Vornüberfallen des Körpers volarwärts ausweichen.

Von grösserem Interesse für unsere Untersuchungen sind die sieben nächsten Beobachtungen, in denen die Verletzung auf eine indirecte Ursache zurückgeführt wird.

Im Fall Neve war das verursachende Moment Sturz auf den Rücken der gebeugten Hand; wäbrend die Hand bereits durch die Unterlage fixirt war, glitt die Ulna, getrieben von der Last des fallenden Körpers, dorsalwärts an ihr vorbei; dagegen hielt die straffe Bandverbindung den Radius in Contact mit dem Carpus.

In den Beobachtungen von Stierlin und White $(3,6)$ wurde die Hand ron einem über eine Rolle laufenden Riemen resp. Strick erfasst und so der Arm gedreht; im Falle Stierlin nach aussen, bei White fehlen nähere Angaben über die Richtung. Ich halte es nicht für glaubhaft, dass gewaltsame Aussendrehung ein volares Heraustreten der Ulna bewirken soll. Ebensowenig wahrscheinlich erscheint mir die Angahe Weiz', dass 'Zug an der supinirten Hand denselben Effect gehabt haben soll. Wenn man bedenkt, wie schwer, fast unmöglich es häufig ist, nach plötzlichen Verletzungen eine auch nur annähernd genaue Anamnese zu erheben, so wird man wohl auch hier einen Irrtum in den anamnestischen Angaben nicht ausschliessen können.

Im Weiz'schen Falle (4) kann meiner Veinung nach nur directe Gewalteinwirkung in Betracht kommen. Wenn bei fixirtem Vorderarm die Hand von einem schweren Körper getroffen und dadurch supinirt wird, so wird doch die Hand von der Ulna volarwärts fortgedrängt, während die Ulna dorsal stehen bleibt. Es ist mir unbegreiflich, wie auf diese Weise eine volare Luxation im unteren Radioulnargelenk entstehen soll; es muss und wird stets, falls eine derartige Verletzung überhaupt wirksam ist, eine dorsale Verrenkung. eintreten. Ich möchte glauben, dass bei jener Frau nicht die Hand, sondern die Ulna von dem fallenden Brett getroffen und so vorgestossen worden ist, demnach also eine directe Entstehungsursache vorliegt.

Die von Leisten dorfer (12) beschriebene Verrenkung muss man sich so entstanden denken, dass, während die Hand vom Brustkorb 
fest an den Voltigirbock gepresst wurde, der sich überschlagende Körper den Vorderarm eine Drehung im supinirenden Sinne um die Hand als festen Punkt ausfiuhren liess. Es kommt hier also der Mechanismus in Frage, den ich oben an zweiter Stelle genannt habe. Die Aussendrehung des Unterarms bei festgestellter Hand, nicht aber Supination der Hand bei fixirtem Vorderarm.

Zuletzt möchte ich noch die Mittheilung von Makeig Jones (10) besprechen; sie ist für mich von besonderem Werthe, da der Verfasser auf Grund einer sehr genauen Anamnese eine völlig mit meiner Untersuchung übereinstimmende Aetiologie anführt: Ein Bergarbeiter wird von dem Bolzen einer Bohrmaschine heftig auf den Handrücken geschlagen, und die Hand dadurch stark pronirt, bei gleichzeitig fixirtem Vorderarm; die Folge ist eine volare Luxation des Ulnaköpfchens. Entgegengesetzt der Lehre Malgaigne's, der volare Verrenkung auf Supination zurückführt, bezeichnet er im vorliegenden Falle die Pronation als ursächlichen Factor; mit vollem Recht, denn durch Innendrehung wird die Hand dorsalwärts ron der Ulna entfernt. Damit ist auch klinisch, wenigstens in einem gut beobachteten Falle, der Beweis für die Richtigkeit meiner Behauptung erbracht.

Fasse ich kurz die Ergebnisse dieser Arbeit zusammen, so möchte ich Folgendes hervorheben:

Als Ursache für die Luxation im unteren Radioulnargelenk muss an erster Stelle die directe Gewalt genannt werden.

Die bisherige Annahme, dass die volare Dislocation durch extreme Supination, die dorsale durch Pronation der Hand zu Stande komme, lässt sich nach der experimentellen Nachprüfung nicht halten. Eine Drehung, die die Hand angreift, kann, wenn sie überhaupt wirksam ist, nur im umgekehrten Sinne wirken. Vielmehr wird die indirecte Gewalt hauptsächlich dann in Betracht kommen, wenn die luxirende Bewegung vom Vorderarm ausgeführt wird, während die Hand den festen Stützpunkt bildet, um den die Drehung stattfindet. Wenn es auch nicht gelungen, experimentell auf diesem Wege die Verrenkung. hervorzurufen, so spricht doch für diese Art der Entstehung, die, wenn auch geringe klinische Erfahrung. Wahrscheinlich spielen daneben noch andere Momente, in erster Linie directer Druck, eine Rolle, die den Mechanismus compliciren. Darauf deutet unter Anderem der negative Ausfall der Leichenversuche. 


\section{Literaturverzeichniss.}

1) Malgaigne, Verrenkungen. 1856.

2) Tillmanns, Archiv f. Heilkunde. 18 i4.

3) Purdom, Edinbourg Medical Journal. 1874.

4) Ohlemann, Archiv f. Chirurgie. 1875.

5) Weir, Archiv of klin. Surgery. 1877. II.

6) Hönigschmidt, Deutsche Zeitschr. f. Chirurgie. X. 1978.

7) Neve, Lancet 1881. II.

8) Stierlin, Schweizer Corresp. Blatt 22. 1989.

9) Briggs, Medical Record N.-Y. 1591.

10) Horrocks, Lancet 1891. I.

11)' Makeig Jones, Lancet 1893. I.

12) White, Brit. Med. Journal. 1893.

13) Kirkby-Thomas, Brit. Med. Journal. 1596. II.

14) Leistend orfer, Dentsche Militärarztl. Zeitschr. 1896. 